\title{
Specific and Somatotopic Functional Magnetic Resonance Imaging Activation in the Trigeminal Ganglion by Brush and Noxious Heat
}

\author{
David Borsook, ${ }^{\star}$ Alexandre F. M. DaSilva, ${ }^{\star}$ Alex Ploghaus, and Lino Becerra ${ }^{\star}$ \\ Center for Pain Functional Neuroimaging and Therapy Research, Athinoula Martinos NMR Center, Department of Radiology, Massachusetts General \\ Hospital, Harvard Medical School, Boston, Massachusetts 02129
}

\begin{abstract}
We used functional magnetic resonance imaging (fMRI) to assess activation in the trigeminal ganglion during innocuous mechanical (brush) and noxious thermal $\left(46^{\circ} \mathrm{C}\right)$ stimulation of the face within the receptive fields of each of the three divisions of the trigeminal nerve in healthy volunteers. For both stimulus types, we observed signal changes only in the ipsilateral ganglion, and activation occurred somatotopically, as predicted by the known anatomical segregation of the neurons comprising the ophthalmic (V1), maxillary (V2), and mandibular (V3) divisions of the nerve. Signal decreased after brush stimuli and increased after the application of noxious heat. The abilities to detect somatotopic activation within the ganglion and to segregate non-noxious mechanical from noxious thermal stimuli suggest that fMRI will be valuable for measuring changes in the trigeminal ganglion in human models of neuropathic pain and in the clinical condition itself and may also be useful in the evaluation of pain therapies.
\end{abstract}

Key words: trigeminal ganglion; human; fMRI; pain; brush; heat

\section{Introduction}

Under normal conditions, primary afferent nerves located in the DRG convey pain information to the CNS. After peripheral inflammation or nerve damage, there are significant anatomical and functional changes within these neurons that contribute to clinical pain states (Snider and McMahon, 1998; Woolf and Salter, 2000; Julius and Basbaum, 2001). Whereas investigations of such changes in the DRG are easy to perform in animal models, the opportunity to obtain DRG tissue from humans is limited (Taub et al., 1995; Wilkinson and Chan, 2001).

The trigeminal ganglion (TG), the equivalent of the dorsal root ganglia, is located at the base of the brain in the posterior cranial fossa across the superior border of the petrous temporal bone. It comprises sensory neurons from the ophthalmic (V1), maxillary (V2), and mandibular (V3) divisions of the trigeminal nerve and is found within Meckel's cave, which is formed by an invagination of the dura mater. The ganglion is crescent shaped and has some somatotopic organization related to the afferent projections from each division. Thus, the structure is fixed in position and has specific landmarks, definable on magnetic resonance imaging (MRI). In the human, it contains $\sim 27,000$ neurons and the associated supporting non-neuronal cells (100 per neuron) (LaGuardia et al., 2000). In rats, the neurons involved in transmission of thermal pain ( $\mathrm{C}$ and $\mathrm{A} \delta$ fibers) account for 60

\footnotetext{
Received March 10, 2003; revised June 19, 2003; accepted July 2, 2003.

${ }^{*}$ D.B., A.F.M.D., and L.B. contributed equally to this work.

Correspondence should be addressed to Dr. David Borsook, Descartes Therapeutics, Inc., 790 Memorial Drive, Cambridge, MA 02139. E-mail: dborsook@dtrx.com.

Copyright $\odot 2003$ Society for Neuroscience $\quad$ 0270-6474/03/237897-07\$15.00/0
}

$70 \%$ of the neurons within the ganglion. Although the minimal number of neurons required for functional activation in the brain is unknown, the concentration of neurons within the TG, its fixed anatomy (i.e., not altered by cardiac or respiratory pulsations), and a pattern of vascularization similar to that seen in the CNS (Smoliar, 1978; Smoliar et al., 1998, 1999) make it a likely target for functional imaging. Thus, a specific unambiguous region of interest (ROI) can be defined anatomically and functionally.

Recent advances in functional neuroimaging have allowed for noninvasive measures of neuronal activation by sensory stimuli, including pain (Becerra et al., 2001; Ploghaus et al., 2000; Davis et al., 2002). However, such applications have been limited to the CNS. Our group has previously reported activation in the trigeminal nucleus after thermal pain applied to the three divisions of the trigeminal nerve (DaSilva et al., 2002). Using the same experimental group, we report that ipsilateral noxious stimulation in the face produces functional MRI (fMRI) activity within the ipsilateral TG.

\section{Materials and Methods \\ Human subjects}

Nine healthy right-handed, male volunteers (mean age, $29.4 \pm 5.05$ years) were recruited for the experiment, which was approved by the Subcommittee on Human Studies at the Massachusetts General Hospital. They had no history of significant dental or facial pain, were not on any medication, and were instructed not to consume caffeine beginning the night before the experiment.

The research investigators explained the experimental protocol to the subjects in detail, including the nature of the research, the temporal sequence, the device used for thermal pain stimulation, and how to rate their pain (Likert's Visual Analogue Scale). During the functional scans, subjects were instructed to keep their heads still and eyes closed. Subjects 

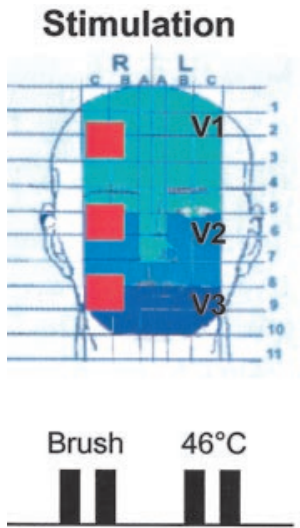

Anatomy of Ganglion

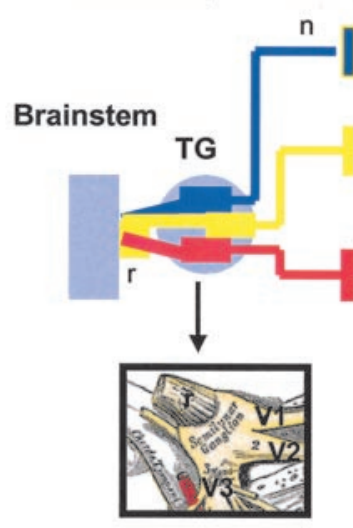

TG Activation

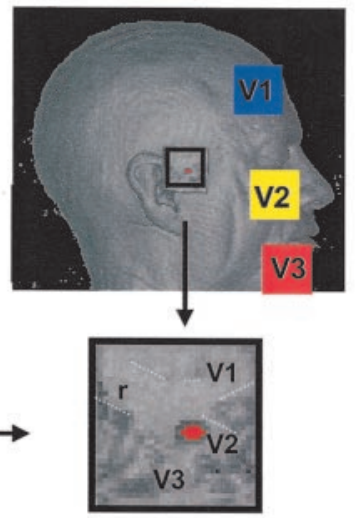

\section{Predicted Activation}

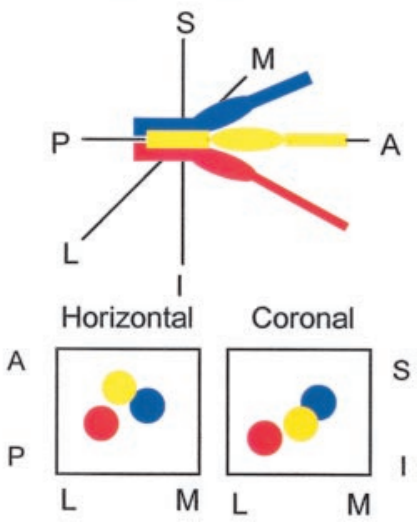

Figure 1. Experimental design, anatomy, and predicted activation in TG. Left, Brush and pain stimuli were applied to regions (indicated by red squares) within the receptive fields of each of the three divisions (V1, V2, and V3) of the trigeminal nerve. Note that stimuli were applied only to the right side of the face. Brush stimuli were administered before thermal stimuli $\left(46^{\circ} \mathrm{C}\right)$. Center left, Diagrammatic representation of the TG and the ophthalmic (V1, blue), maxillary (V2, yellow), and mandibular (V3, red) nerves. The neuronal bodies of the nerves are segregated somatotopically within the ganglion, as indicated by the small boxes for each nerve. The central processes of $\mathrm{TG}$ neurons ( $\mathrm{r}$, dorsal roots) project to central terminations within the trigeminal nuclear complex (spV) of the brainstem. Center right, $A$ three-dimensional reconstruction of the right side of the face of a subject stimulated in the V2 region is shown with a window cut out to view the TG. A box of this view is shown in the enlarged view below. Note that activation can be observed within the V2 distribution of the ganglion. Right, The relative ( $x, y$, and $z$ ) positions of the V1 (blue), V2 (yellow), and V3 (red) divisions of the trigeminal nerve within the trigeminal fossa. Below this, the locations of predicted activations in the TG after stimulation of each division are shown in the corresponding color in the coronal and horizontal planes.

could halt the experiment at any time by activating a safety mechanism held in one hand.

\section{Experimental paradigm and analysis}

Subjects received innocuous mechanical (brush) and noxious thermal $\left(46^{\circ} \mathrm{C}\right)$ stimuli to premarked areas within the receptive fields of the V1, V2, and V3 (Fig. 1). The different stimuli were administered to identical areas on separate trials (i.e., brush to V1, V2, and V3, followed by thermal stimuli in the same order), and fMRI data were acquired as individual functional data sets. The TG was visualized within the acquired brain slices, as described previously (DaSilva et al., 2002). Each of the three divisions of the trigeminal nerve contributes to the TG. The anatomical definition of the ganglion and the segregation of afferent fibers into three distinct components within the ganglion predicted somatotopic activation within the ganglion (Fig. 1).

\section{Sensory stimulation}

Mechanical (brush). Mechanical stimuli were applied sequentially, in separate fMRI acquisitions, to each of the three divisions of the trigeminal nerve in the same region (premarked) that the heat probe was applied later. Stimulation was applied within a $1.6 \times 1.6 \mathrm{~cm}$ premarked area of the skin (the identical area used for thermal stimulation). Using a brush attached to a mechanical transducer designed for use in the magnet, brush stimuli were applied with a frequency of $1-2 \mathrm{~Hz}$. Continuous 25 sec brush stimuli were applied four times with an interstimulus interval of $30 \mathrm{sec}$. Brush was not alternated with heat because the latter could sensitize the skin.

Thermal $\left(46^{\circ} \mathrm{C}\right)$. The thermal pain stimulation was applied to a premarked site of each division of the right trigeminal nerve using a $1.6 \times 1.6$ $\mathrm{cm}$ Peltier thermode (Medoc, Haifa, Israel). Each site received two $46^{\circ} \mathrm{C}$ stimuli lasting $25 \mathrm{sec}$ each, separated by three $30 \mathrm{sec}$ baseline stimuli $\left(32^{\circ} \mathrm{C}\right)$.

\section{Scanning}

A Siemens (Erlangen, Germany) Sonata System 1.5T was used for the research. After a three-plane scout scan, the axial and coronal scouts were used for the placement of the three-dimensional anatomical sagittal scan. Functional images were prescribed with 45 time points of 30 slices, each with $3 \mathrm{~mm}$ slice thickness, oriented parallel to the medulla in an oblique plane (repetition time/echo time, $3.5 \mathrm{sec} / 40 \mathrm{msec}$; in-plane resolution, $3.125 \mathrm{~mm}$ ), including the middle portion of the forebrain, brainstem, and TG.

\section{Data analysis}

Functional data were processed using MEDx 3.3 (Sensor Systems, Bethesda, MD). The following sequence was used: (1) rigid-body motion correction for functional scans, in which data sets with $>1 \mathrm{~mm}$ motion were discarded; (2) spatial filtering with an isotropic Gaussian kernel of 6 $\mathrm{mm}$ width for Talairach group analysis and $1.5 \mathrm{~mm}$ in-plane for native (non-Talairach) individual analysis; and (3) baseline intensity normalization.

Both anatomical and functional native data were also transformed into the Talairach coordinate system (Talairach and Tournoux, 1988) for average analysis of individual data.

Voxel-by-voxel Student's $t$ test analysis was performed to compare noxious thermal stimulus $\left(46^{\circ} \mathrm{C}\right)$ with baseline period $\left(32^{\circ} \mathrm{C}\right)$. Resulting $\mathrm{z}$ maps were translated into $-\log P$ maps. These maps were used to color code intensity of activation. These maps were then transformed into Talairach space for localization of the ROI. The individual Talairach activation was validated only if located within three pixels from the average group peak activation coordinates.

\section{Results}

\section{Anatomical localization}

Anatomically, the anterior border of the TG is delimited by the superior orbital fissure for the ophthalmic extension and by the foramen rotundum for the maxillary extension and foramen ovale for mandibular extension. The location of the TG was defined in each individual by tracing the nerve from its roots as it enters the pons and similarly in the group data. Serial sections of standard MRI images of the base of the brain were used as shown (Fig. 2). First, the emergence of the trigeminal root from the midlateral surface of the pons was defined. The trigeminal root was followed up to Meckle's cave, in the floor of the middle cranial fossa, where the TG sits (Fig. 2a,g, dotted circles).

\section{Psychophysical ratings}

After each stimulus, subjects rated their pain using the visual analog scale (VAS). No pain was reported after the brush stimuli. The average pain scores for the thermal pain stimuli were $6.2 \pm$ $1.0(n=6)$ for stimuli to the V1 area, $6.6 \pm 0.6(n=7)$ for V2, and $5.5 \pm 1.0(n=5)$ for V3. No significant differences between VAS 

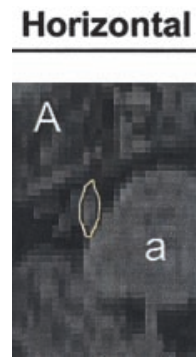

\section{Coronal}
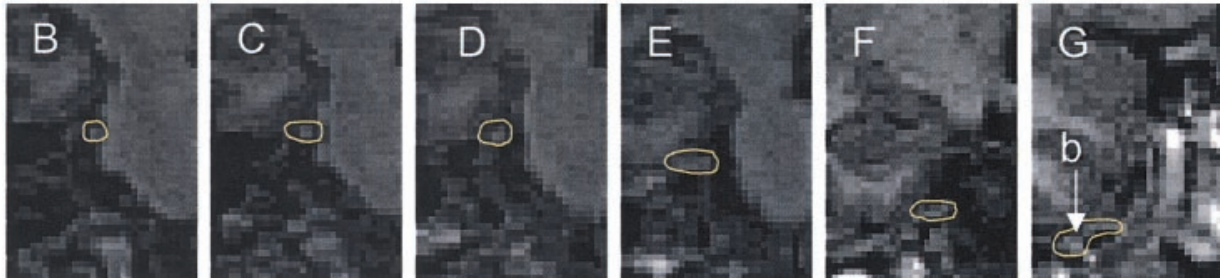

Figure 2. Method for localizing the TG. Serial sections of standard T-2-weighted images of the base of the brain demonstrate the method used to determine the location of the TG within the middle cranial fossa. The emergence of the trigeminal root from the midlateral surface of the pons (a) was defined ( $A$, dotted circle). The trigeminal root was then followed until Meckle's cave ( $B-G$, dotted circle), located in the floor of the middle cranial fossa (b, arrow), in which the TG is formed. Additional anatomical landmarks used included the superior orbital fissure (which delimits the anterior border of the TG for the ophthalmic extension), the foramen rotundum (for the maxillary extension), and foramen ovale (for the mandibular extensions).

Brush

\section{Opthalmic}

(V1)

Maxillary

(V2)
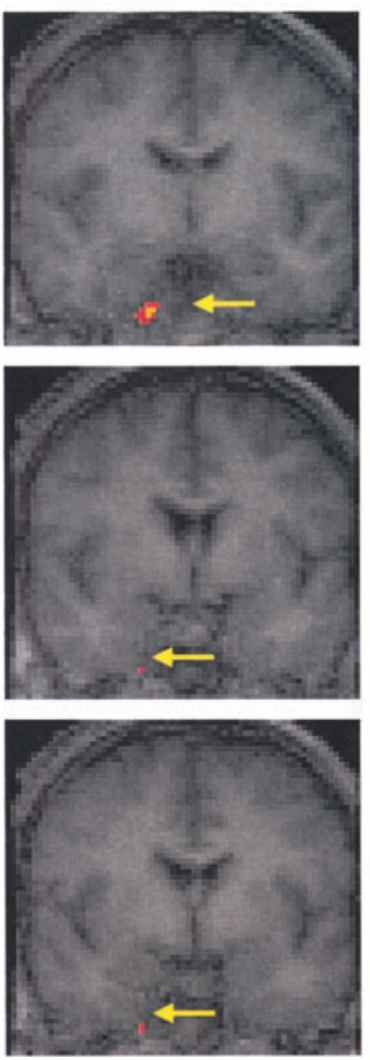

Horizontal
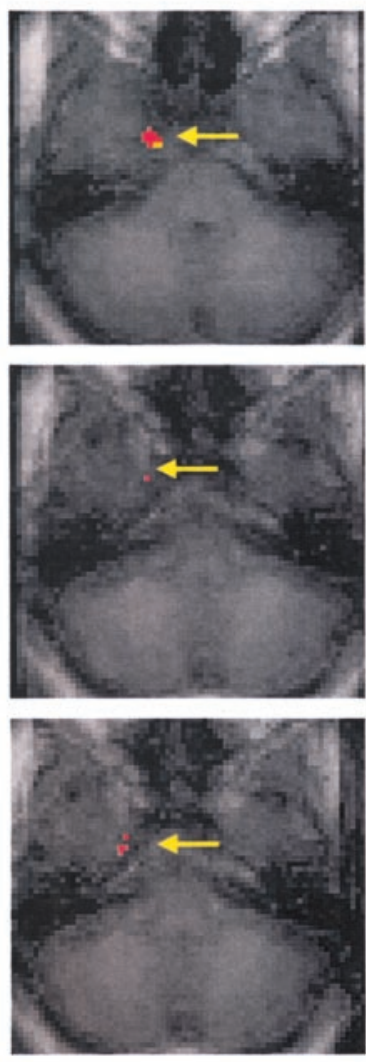

\%Signal change
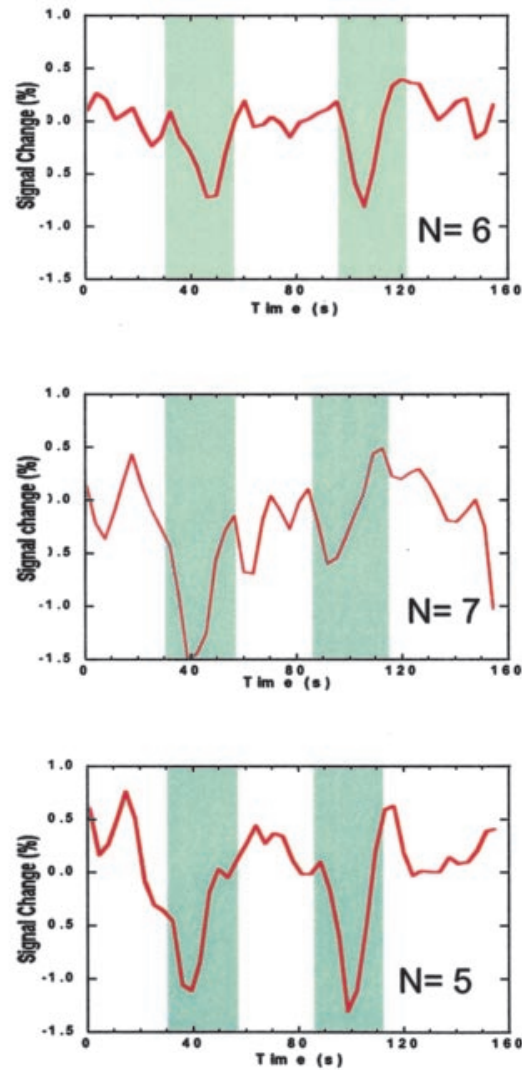

Figure 3. Activation in the TG in response to brush stimuli to the face. Average statistical maps and time course analysis within the ophthalmic (V1), maxillary (V2), and mandibular (V3) divisions of the TG after brush stimuli. Coronal and horizontal views of activations are shown. Arrows indicate activation. Activations are time-locked with the stimulus presentation, as shown by the gray bars. Time courses are shown as relative signal change ( $y$-axis) versus time in seconds ( $x$-axis).

scores between each division were observed (Student's $t$ test). Note that these data were derived from the same subjects included in the fMRI analysis and have been reported previously.

\section{fMRI activation in the three divisions of the TG after} sensory stimuli

Data from two of the nine subjects scanned were eliminated from further evaluation because of movement artifact.

When group fMRI data were averaged, activation was observed in coronal and horizontal planes in the right TG after both innocuous mechanical and noxious thermal stimuli to the right side of the face within the receptive fields for V1, V2, and V3 (Figs.
3, 4; Table 1). Intriguingly, the polarity of signal change is opposite: a negative signal change is observed after brush (Fig. 3), and a positive signal change is observed after noxious heat (Fig. 4). The activations seen after noxious thermal stimulus to the ophthalmic and mandibular divisions were less significant than that for the maxillary division. No activation was observed in the contralateral (left) trigeminal nucleus after any of the six stimuli (three brush and three thermal) in any subject.

To confirm that the group data were not biased by activations in a few individuals, data from each individual were analyzed using both the Talairach system and native images (see Materials and Methods). Individual activation was validated only if it was 
Heat

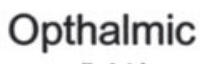

(V1)

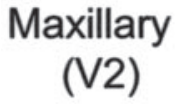

Mandibular (V3)
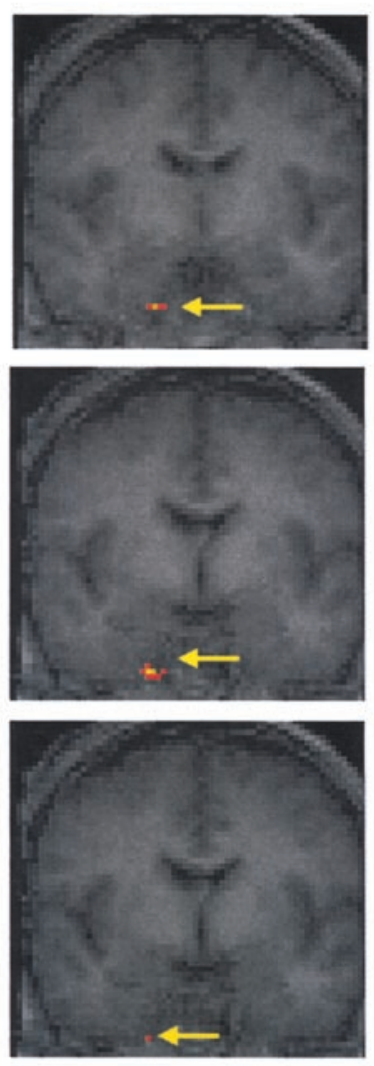

Horizontal
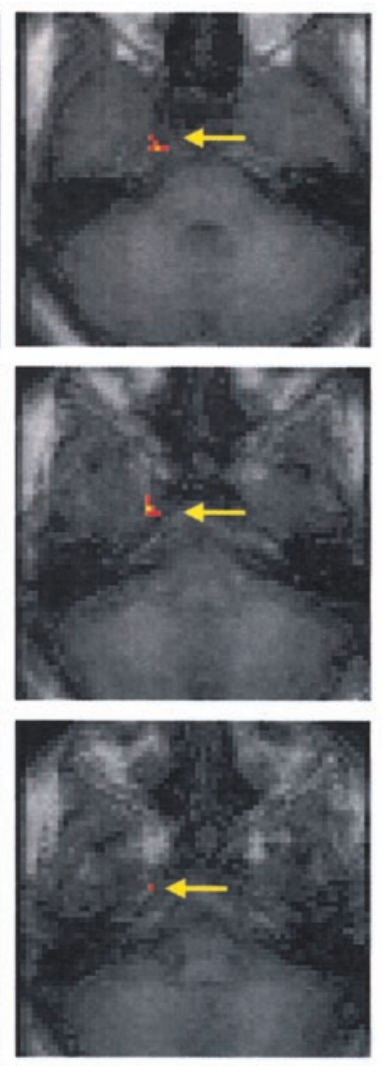

\%Signal change
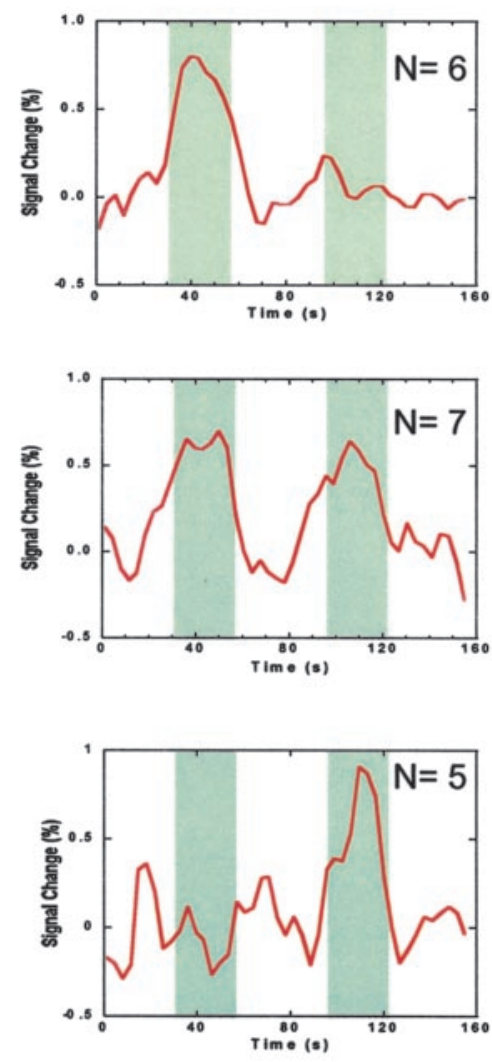

Figure 4. Activation in the TG in response to thermal stimuli to the face. The figures show statistical maps and time course analysis of activations within the ophthalmic (V1), maxillary (V2), and mandibular (V3) divisions of the TG after $46^{\circ} \mathrm{C}$ stimuli. The statistical maps show activation within the inferior portion of the middle cranial fossa. Coronal and horizontal views of activations are shown (arrows). Activations corresponded with the stimulus presentation, as shown by the gray bars. Time courses are shown as relative signal change ( $y$-axis) versus time in seconds ( $x$-axis).

Table 1. Group analysis: brush

\begin{tabular}{|c|c|c|c|c|c|}
\hline \multirow[b]{2}{*}{ Stimulus site } & \multicolumn{3}{|c|}{ Talairach coordinates } & \multirow{2}{*}{$\begin{array}{l}\text { Volume } \\
\left(\mathrm{cm}^{3}\right)\end{array}$} & \multirow[b]{2}{*}{$p$} \\
\hline & $\mathrm{ML}(X)$ & $\operatorname{AP}(Y)$ & $\mathrm{SI}(Z)$ & & \\
\hline Ophthalmic division (V1) & 16 & -8 & -34 & 0.18 & $4.2 \times 10^{-4}$ \\
\hline Maxillary division (V2) & 20 & -2 & -36 & 0.02 & $3.9 \times 10^{-2}$ \\
\hline Mandibular division (V3) & 20 & -8 & -34 & 0.07 & $1.5 \times 10^{-2}$ \\
\hline
\end{tabular}

located within three pixels of the average peak coordinates. For the brush stimuli, activation was seen for six of seven subjects in V1, V2, and V3 (Table 2). For heat stimuli, activation was seen for six of seven subjects in V1, all subjects from V2, and five of seven subjects in V3 (Tables 3, 4).

Examples of individual activations are shown for brush and for heat (Fig. 5). The activation maps shown have been masked to include activation within the inferior portion of the middle cranial fossa on each side. The delimited area corresponds to a volume of $7.73 \mathrm{~cm}$ (Woolf and Salter, 2000), embracing both TG and adjacent structures on each side. Note that there is no neuronal activation in the contralateral TG.

We mapped the activations observed after stimulation of each division with brush or heat onto a single map (Fig. 6). As predicted based on its anatomy (Fig. 1), activation in the TG after both stimulus types was somatotopically arranged.

\section{Discussion}

Currently, routine methods for noninvasive evaluation of pain states or pain therapies that have applications in the clinic are still in development. Here, we provide evidence that activity in the TG can be imaged using fMRI, suggesting that fMRI of the TG can provide an objective marker of facial pain, a method for evaluating the efficacy of analgesics in human pain that can be easily integrated with standard drug assessment techniques and a method suitable for longitudinal evaluation of pain-induced changes within the PNS.

The trigeminal nerve contains both motor and sensory fibers. The primary afferent sensory fibers of all types $(\mathrm{A} \beta, \mathrm{A} \delta$, and $\mathrm{C})$ have their neuronal bodies within the TG. Large myelinated fibers $(A \beta)$ convey a number of sensations including light touch, whereas unmyelinated $\mathrm{C}$ and $\mathrm{A} \delta$ fibers primarily convey nociceptive information. A large percentage of trigeminal neurons are involved in pain processing. Extracellular recordings in monkeys revealed activation in TG neurons after thermal stimuli at 3849 ${ }^{\circ} \mathrm{C}$ (Beitel and Dubner, 1976; Croze et al., 1976). Maximum discharge frequencies were obtained in the noxious heat range $\left(>44^{\circ} \mathrm{C}\right)$. Experiments have correlated the activation of warm and nociceptive $\mathrm{C}$-fiber afferents in the monkey with human 
Table 2. Individual analysis: brush

\begin{tabular}{|c|c|c|c|c|c|c|c|c|}
\hline \multirow[b]{2}{*}{ Stimulus site } & \multicolumn{7}{|l|}{ Subject } & \multirow[b]{2}{*}{ Total } \\
\hline & 1 & 2 & 3 & 4 & 5 & 6 & 7 & \\
\hline Ophthalmic division (V1) & $-/(-)$ & $+/(+)$ & $+/(+)$ & $+/(+)$ & $+/(+)$ & $+/(+)$ & $\Delta$ & $5 /(6)$ \\
\hline Maxillary division (V2) & $-/(-)$ & $+/(+)$ & $+/(+)$ & $-/(-)$ & $+/(+)$ & $-/(+)$ & $\Delta$ & $4 /(6)$ \\
\hline Mandibular division (V3) & $-/(-)$ & $+/(+)$ & $-/(-)$ & $+/(+)$ & $+/(+)$ & $+/(+)$ & $\Delta$ & $4 /(6)$ \\
\hline
\end{tabular}

+ , Activation; - , no activation; Talairach $\leftarrow \pm /( \pm) \rightarrow$ anatomic; $\Delta$, movement.

Table 3. Group analysis: thermal

\begin{tabular}{|c|c|c|c|c|c|}
\hline \multirow[b]{2}{*}{ Stimulus site } & \multicolumn{3}{|c|}{ Talairach coordinates } & \multirow[b]{2}{*}{ Volume $\left(\mathrm{cm}^{3}\right)$} & \multirow[b]{2}{*}{$p$} \\
\hline & $\operatorname{ML}(X)$ & $\operatorname{AP}(Y)$ & $\operatorname{SI}(Z)$ & & \\
\hline Ophthalmic division (V1) & 20 & -6 & -30 & 0.22 & $1.0 \times 10^{-3}$ \\
\hline Maxillary division (V2) & 20 & -4 & -34 & 0.38 & $2.5 \times 10^{-5}$ \\
\hline Mandibular division (V3) & 20 & -4 & -38 & 0.01 & $3.6 \times 10^{-2}$ \\
\hline
\end{tabular}

Table 4. Individual analysis: thermal

\begin{tabular}{|c|c|c|c|c|c|c|c|c|}
\hline \multirow[b]{2}{*}{ Stimulus site } & \multicolumn{7}{|l|}{ Subject } & \multirow[b]{2}{*}{ Total } \\
\hline & 1 & 2 & 3 & 4 & 5 & 6 & 7 & \\
\hline Ophthalmic division (V1) & $-/(-)$ & $+/(+)$ & $+/(+)$ & $+/(+)$ & $+/(+)$ & $+/(+)$ & $\Delta$ & $5 /(6)$ \\
\hline Maxillary division (V2) & $+/(+)$ & $-/(+)$ & $+/(+)$ & $-/(-)$ & $+/(+)$ & $+/(+)$ & $+/(+)$ & $6 /(7)$ \\
\hline Mandibular division (V3) & $-/(+)$ & $\theta$ & $\theta$ & $-/(-)$ & $+/(+)$ & $+/(+)$ & $-/(-)$ & $3 /(5)$ \\
\hline
\end{tabular}

+ , Activation; - , no activation; Talairach $\leftarrow \pm /( \pm) \rightarrow$ anatomic; $\Delta$, movement; $\ominus$, machine malfunction.

psychophysical measures (LaMotte and Campbell, 1978). Our experiments used a thermal stimulus of $46^{\circ} \mathrm{C}$, well above the activation threshold of nociceptors, and subjects reported significant pain with this stimulus (VAS scores $>5 / 10$ ), strongly supporting the activation of $\mathrm{C}$ fibers by this stimulus.

The application of either brush or thermal stimuli to the V1, $\mathrm{V} 2$, or V3 divisions of the face produced fMRI activation within the ipsilateral TG in seven healthy volunteers. Two of nine subjects were excluded because of motion artifact. Activation was present in six of seven subjects for brush for all divisions and between five and seven of seven subjects for thermal stimuli (depending on division stimulated; see Results). Signal change in the order of $0.4-1.5 \%$ was observed in these cases. No activation was seen in the contralateral TG in any subject, suggesting that these activations were caused by the stimuli and are not artifacts.

Each of the three divisions of the trigeminal nerve consists of processes from neurons with cell bodies in the TG. The neuronal bodies for both large $(\mathrm{A} \beta)$ and small $(\mathrm{C}$ and $\mathrm{A} \delta$ ) fibers are arranged segmentally within the TG. Cell bodies of the mechanoreceptive and nociceptive afferents of the ophthalmic division (V1) are found medially and anteriorly, those of the mandibular division (V2) are caudal and lateral, and those from the maxillary division are present in between (Oyagi et al., 1989). Thus, the somatotopic activation patterns that we observed for both brush and thermal pain correspond to the anatomical formulation of the ganglion, as expected.

Functional imaging of the TG has not been reported before, and a number of issues need to be considered. These include the fixed location of the ganglion, vascularization, and the number of neurons responding within the TG.

The trigeminal nerve is the largest and most complex of the 12 cranial nerves and also the largest DRG in the body (Jannetta, 1967; Brown, 1997; Shankland, 2000). It is located at the base of the brain in the posterior cranial fossa within Meckel's cave. It is, thus, in a fixed position with clearly marked anatomical features, easily recognized by MRI. In addition, as noted in Materials and Methods, anatomical scans (Kamel and Toland, 2001) were used to trace the dorsal root fibers entering the brainstem back to the
TG. The roots start along the ventral surface of the brainstem at the midpontine level and are easily defined by their size and location. The presence of anatomical markers clearly visible on fMRI allows us to be confident of our localization of the TG when analyzing the specificity of activation.

The blood supply to the trigeminal ganglion originates from the internal carotid artery via the cavernous sinus (Krisht et al., 1994). The microcirculatory bed in the TG has been studied anatomically (Smoliar, 1978; Smoliar et al., 1998, 1999). In the internal layers of perineurium, pericapillaries, capillaries, and postcapillaries are present; in the sheaths surrounding the root fibers and in endoneurium, there are only capillaries. Microscopic evaluation of blood vessels within the TG revealed that arteriolovenular anastomoses facilitate blood redistribution within the superficial layers of the trigeminal nerve, and that precapillary sphincters and transepineural arterioles are involved in the regulation of blood flow in deeper layers of the nerve trunk (Smoliar et al., 1998). Together, these data suggest that the vascular structure within the TG is similar to that observed within the CNS and should provide a reliable basis for blood oxygenation leveldependent (BOLD) measures. Currently, we are unaware of data demonstrating segmental or somatotopic changes of blood flow within the TG.

Because the internal carotid artery is located medial to the TG, cardiac pulsation could produce artifacts. These artifacts should appear bilaterally. However, the absence of activation in the contralateral TG indicates that we did not observe it.

The minimal number of neurons that must be activated to produce a signal detectable by fMRI is not known, and the current data add some useful information regarding this issue. The human TG contains $\sim 25,000$ neurons (LaGuardia et al., 2000). This includes all of the sensory neurons innervating the face via the trigeminal nerve. In our experiments, we stimulated a small region of the face, corresponding to $<5-10 \%$ of the total surface area innervated by the ipsilateral trigeminal nerve. Within the group of neurons activated, issues such as frequency of action potentials may be the salient issue in driving measurable BOLD changes. Whatever the underlying basis, the results indicate that 


\section{Individual Activation}

Cor.

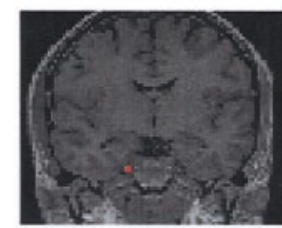

Sag.

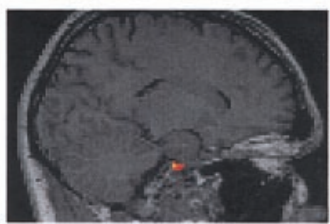

Hor.

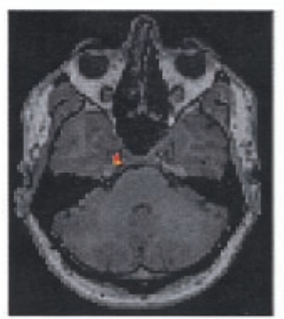

Brush
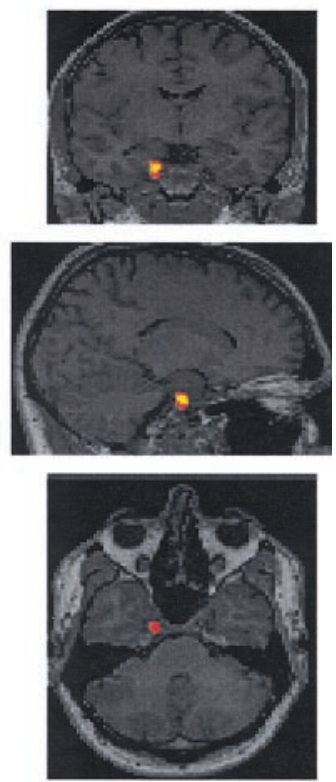

Heat
Figure 5. Individual analysis-TG activation in response to brush and heat. The figures show statistical maps of activations within the maxillary (V2) and division of the TG after brush (left) and noxious heat (right).
Coronal
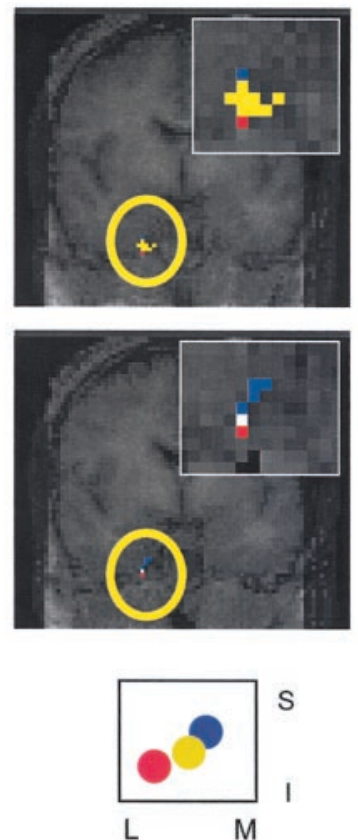

Horizontal
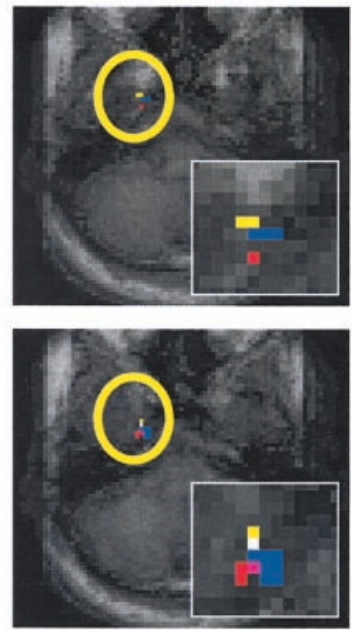

A

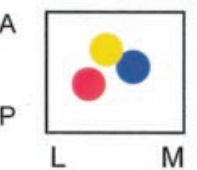

Figure 6. Overlap maps showing activations within the TG for pain and brush. The location within the middle cranial fossa is shown mapped onto coronal and horizontal T-2-weighted MRI images of the brain. The square inset shows an enlargement of each overlap activation for stimulation in V1 (blue), V2 (yellow), and V3 (red). Note how these correspond to the predicted activations in these two planes, shown in the inset at the bottom of the figure (see also Fig. 1, right).

activation within quite small populations of neurons can be measured with BOLD analysis.

Our data show increased BOLD signal in response to noxious heat and decreased signal in response to a brush stimulus. The explanation for this difference in the polarity of signal change may be that these responses take place in separate neural populations. Brush stimuli activate large myelinated $\mathrm{A} \beta$ fibers, whereas noxious thermal heat activates both small unmyelinated (C) and thinly myelinated $\mathrm{A} \delta$ fibers. Whereas the $\mathrm{A} \beta$ fibers exhibit fast conduction velocities $(100 \mathrm{~m} / \mathrm{sec})$ and rapid repriming of sodium currents, $\mathrm{A} \delta$ and $\mathrm{C}$ fibers have slow conducting velocities (5-20 m/sec for A $\delta$ and $0.1-1 \mathrm{~m} / \mathrm{sec}$ for C fibers) and slower repriming of sodium channels (Everill et al., 2001). The response in $\mathrm{A} \beta$ fibers is an on-off response compared with the slower offset of activity in C fibers.

A potential explanation of negative activation to brush but not to heat is provided below. When noxious heat is applied to the periphery, small fibers ( $\mathrm{C}$ and $\mathrm{A} \delta$ ) induce a relatively small number of synaptic events; hence, an initial dip takes place in the BOLD response because flow by itself does not clear out increased deoxyhemoglobin because of activity (Buxton et al., 1998). However, blood flow, and especially increased blood volume, turns the signal around, giving a positive response as a result of the augmented capillary volume diluting the concentration of deoxyhemoglobin, and makes flow more efficient in removing it. Thus, the positive signal is dependent on the capacity to increase volume and flow. In the case of brush stimulation, the large $\mathrm{A} \beta$ fibers produce more synaptic activity, as has been evidenced from electrophysiology experiments (Jacquin et al., 1986; Brown et al., 1987; Steedman and Zachary, 1990). In this case, the required increase in blood flow and volume might not be achieved, and, hence, the negative signal observed may represent an extended initial dip in the BOLD response (Harel et al., 2002). In addition, there may be some effects from sympathetic inputs to the ganglion, and heat and brush have different effects on the sympathetic tone of vessels surrounding the activated neurons (Koltzenburg, 1997). Correlation of activation by heat and brush in the ganglion with activation in the trigeminal nucleus has been defined and is the subject of a separate study (D. Borsook, A. DaSilva, L. Becerra, unpublished observations).

The interpretation of negative signal changes in BOLD signal is still unresolved. The BOLD signal has been correlated with action potentials and slow varying field potentials (Logothetis et al., 2001; Thompson et al., 2003). In this formulation, inhibitory interneurons and dentrites/cell soma are thought to contribute to the signal. The intrinsic TG neurons are bipolar, with no dendrites, and there are no inhibitory interneurons present. The TG does contain sympathetic inputs to the vasculature that may influence neural function. However, the relative structural simplicity of the TG provides a simpler system for interpreting the BOLD response.

\section{Conclusions}

This study demonstrates that fMRI may be used to image activation in the TG after brush stimuli activating $\mathrm{A} \beta$ fibers and thermal stimuli in the painful range (i.e., $>44^{\circ} \mathrm{C}$ ) known to activate both $\mathrm{C}$ and $\mathrm{A} \delta$ fibers. Further development of this approach, together with mapping of central trigeminal pathways, could provide a basis for future studies for objective evaluation of peripheral and central changes in clinical conditions (e.g., postherpetic neuralgia affecting the face, damage to trigeminal nerves after dental surgery) and the efficacy of therapies for facial pain.

\section{References}

Becerra L, Breiter HC, Wise R, Gonzalez RG, Borsook D (2001) Reward circuitry activation by noxious thermal stimuli. Neuron 32:927-946. Beitel RE, Dubner R (1976) Response of unmyelinated (C) polymodal no- 
ciceptors to thermal stimuli applied to monkey's face. J Neurophysiol 39:1160-1175.

Brown AG, Koerber HR, Noble R (1987) Actions of trains and pairs of impulses from single primary afferent fibers on single spinocervical tract cells in cat. J Physiol (Lond) 382:313-329.

Brown JA (1997) The trigeminal complex. Anatomy and physiology. Neurosurg Clin North Am 8:1-10.

Buxton RB, Wong EC, Frank LR (1998) Dynamics of blood flow and oxygenation changes during brain activation: the balloon model. Magn Reson Med 39:855-864.

Croze S, Duclaux R, Kenshalo DR (1976) The thermal sensitivity of the polymodal nociceptors in the monkey. J Physiol (Lond) 263:539-562.

DaSilva AF, Becerra L, Makris N, Strassman AM, Gonzalez RG, Geatrakis N (2002) Somatotopic activation in the human trigeminal pain pathway. J Neurosci 22:8183-8192.

Davis KD, Pope GE, Crawley AP, Mikulis DJ (2002) Neural correlates of prickle sensation: a percept-related fMRI study. Nat Neurosci 5:1121-1122.

Everill B, Cummins TR, Waxman SG, Kocsis JD (2001) Sodium currents of large (A $\beta$-type) adult cutaneous afferent dorsal root ganglion neurons display rapid recovery from inactivation before and after axotomy. Neuroscience 106:161-169.

Harel N, Lee SP, Nagoaka T, Kim DS, Kim SG (2002) Origin of negative blood oxygenation level-dependent fMRI signals. J Cereb Blood Flow Metab 22:908-917.

Jacquin MF, Renehan WE, Mooney RD, Rhoades RW (1986) Structurefunction relationships in rat medullary and cervical dorsal horns. I. Trigeminal primary afferents. J Neurophysiol 55:1153-1186.

Jannetta PJ (1967) Gross (mesoscopic) description of the human trigeminal nerve and ganglion. J Neurosurg 26:109-111.

Julius D, Basbaum AI (2001) Molecular mechanisms of nociception. Nature 413:203-211.

Kamel HA, Toland J (2001) Trigeminal nerve anatomy: illustrated using examples of abnormalities. AJR Am J Roentgenol 176:247-251.

Koltzenburg M (1997) The sympathetic nervous system and pain. In: Handbook of experimental pharmacology (Dickenson AH, Besson JM, eds), pp 61-91. New York: Springer.

Krisht A, Barnett DW, Barrow DL, Bonner G (1994) The blood supply of the intracavernous cranial nerves: an anatomic study. Neurosurgery 34:275-279.
LaGuardia JJ, Cohrs RJ, Gilden DH (2000) Numbers of neurons and nonneuronal cells in human trigeminal ganglia. Neurol Res 22:565-566.

LaMotte RH, Campbell JN (1978) Comparison of responses of warm and nociceptive C-fiber afferents in monkey with human judgments of thermal pain. J Neurophysiol 41:509-528.

Logothetis NK, Pauls J, Augath M, Trinath T, Oeltermann A (2001) Neurophysiological investigation of the basis of the fMRI signal. Nature 412:150-157.

Oyagi S, Ito J, Honjo I (1989) Topographic study of the feline trigeminal ganglion via the horseradish peroxidase tracer method. Brain Res 476:382-383.

Ploghaus A, Tracey I, Clare S, Gati JS, Rawlins JN, Matthews PM (2000) Learning about pain: the neural substrate of the prediction error for aversive events. Proc Natl Acad Sci USA 97:9281-9286.

Shankland II WE (2000) The trigeminal nerve. Part I: an over-view. Cranio 18:238-248.

Smoliar EM (1978) Microcirulatory bed of the human trigeminal nerve. Arkh Anat Gistol Embriol 74:23-27.

Smoliar E, Smoliar A, Sorkin L, Belkin V (1998) Microcirculatory bed of the human trigeminal nerve. Anat Rec 250:245-249.

Smoliar E, Smoliar A, Belkin VS (1999) Innervation of human trigeminal nerve blood vessels. Cells Tissues Organs 165:40-44.

Snider WD, McMahon SB (1998) Tackling pain at the source: new ideas about nociceptors. Neuron 20:629-632.

Steedman WM, Zachary S (1990) Characteristics of background and evoked discharges of mulitreceptive neurons in lumbar spinal cord of cat. J Neurophysiol 63:1-15.

Talairach J, Tournoux P (1988) Co-planar stereotaxic atlas of the human brain. Stuttgart, Germany: Thieme.

Taub A, Robinson F, Taub E (1995) Dorsal root ganglionectomy for intractable monoradicular sciatica. A series of 61 patients. Stereotact Funct Neurosurg 65:106-110.

Thompson JK, Peterson MR, Freeman RD (2003) Single-neuron activity and tissue oxygenation in the cerebral cortex. Science 299:1070-1072.

Wilkinson HA, Chan AS (2001) Sensory ganglionectomy: theory, technical aspects, and clinical experience. J Neurosurg 95:61-66.

Woolf CJ, Salter MW (2000) Neuronal plasticity: increasing the gain in pain. Science 288:1765-1769. 